\title{
Nuclear quadrupole resonance for explosive detection
}

\author{
Resonancia nuclear en cuadrupolo para la detección de explosivos \\ Lorena Cardona $^{1} \quad$ Jovani Jiménez $^{1} \quad$ Nelson Vanegas $^{1}$ \\ Recibido 26 de marzo de 2013, aceptado 30 de septiembre de 2014 \\ Received: March 26, $2013 \quad$ Accepted: September 30, 2014
}

\begin{abstract}
Nuclear quadrupole resonance (NQR) is a spectroscopic technique that can detect explosives of high chemical specificity and therefore it is very suitable for the landmine detection problem. There are several factors affecting the relation of noise signal ratio with NQR: molecular dynamics of relaxation, interference signals, thermal noise, explosive amount, distance, temperature and the design of the detecting system. Literature has searched ways for solving one or more of these factors, but since not all the work has been focused on landmine detection, many have only been tested in simulation or with data obtained under controlled laboratory conditions, where the detection is not remote (there is no separation between the sample and the detection system). This paper makes a review of the state of art in NQR, analyzing its relevance to the landmine detection problem and concluding about unsolved problems that could be the focus of future research.
\end{abstract}

Keywords: Nuclear quadrupole resonance, landmine detection, explosive detection, landmine, demining.

\section{RESUMEN}

La resonancia nuclear en cuadrupolo (NQR) es una técnica espectroscópica que permite detectar explosivos con alta especificidad química, y por esta razón es muy adecuada para el problema de detección de minas. Hay varios factores que afectan la relación señal ruido obtenida con esta tecnología: la dinámica de relajación molecular, las señales de interferencia, el ruido térmico, la distancia a la que se encuentra el explosivo, su cantidad, temperatura y el diseño del sistema de detección. En la literatura se encuentran trabajos enfocados en resolver uno o más de estos factores, pero ya que no todos los trabajos están enfocados en la detección de minas, muchos solo se han probado en simulación o con datos obtenidos bajo condiciones controladas de laboratorio donde la detección no es remota (no hay separación entre la muestra y el sistema de detección). Aquí se hace una revisión del estado del arte en la NQR, analizando su pertinencia con el problema de detección de minas terrestres y mostrando áreas no resueltas en el tema que pueden ser objeto de futuras investigaciones.

Palabras clave: Resonancia nuclear en cuadrupolo, detección de minas, detección de explosivos, minas antipersona, desminado.

\section{INTRODUCTION}

Landmine detection by detecting the explosive charge is probably the ideal way to differentiate a landmine of its surroundings. Other techniques that detect the landmine case suffer of high false alarm rate, being this a current problem found in the landmine detection literature [1].

1 Departamento de Ciencias de la Computación y la Decisión. Universidad Nacional de Colombia. Carrera 80 No 65-223, Of. M8A-309. Medellín, Colombia. E-mail: lcardon0@unal.edu.co; jajimen1@unal.edu.co; nvanegas@unal.edu.co 
Chemical and biological methods, based on the detection of explosive traces, suffer significantly due to moisture, soil pollution sensor saturation and low concentrations of explosive traces [2].

Besides, nuclear quadrupole resonance (NQR) is a specific chemical sensing method that does not require the transfer of chemicals from the mine to the sensor. NQR works by sending radiofrequency (RF) waves into the ground and measuring the returning signal that only occurs if high explosive concentration is present, meaning that explosive traces, present in post-war conditions, do not cause false alarms.

This paper makes a review of the state of the art of NQR, starting with an explanation of the working principle of this technique and the problems encountered for its implementation.

\section{CHARACTERISTICS OF NQR}

NQR is a spectroscopy technique very similar to nuclear magnetic resonance, which exploits the fact that some nuclei possess electric quadrupole moment. This moment is a measure of how the nuclear charge distribution deviates away from spherical symmetry and it happens in nuclei with greater spin or equal to $1[3]$.

The interaction of the quadrupole moment with the electric field gradient at the nuclei causes some nuclear orientation states to be more energetic than the others. Energy states can be excited by RF pulses whose frequency matches the energy difference between two states. The pulses disturb the nuclei orientations and as they have magnetic moment, their movement induces a voltage in a detection coil [4].

When NQR is used to detect explosives, attention is concentrated in ${ }^{14} \mathrm{~N}$ nuclei since this element is present in most explosives, has a natural abundance of $99.63 \%$ and its spin is equal to 1 . Because energy difference between ${ }^{14} \mathrm{~N}$ nuclei states depends on the gradient of local electric field, which in turn depends on the atomic bonds and molecule structure, the frequencies required for ${ }^{14} \mathrm{~N}$ nuclei excitation vary according to the compound. That is why NQR has high chemical specificity.
Each compound has more than one excitation frequency depending on the magnitude of the spin, since it determines the number of energy levels (which is 3 for spin 1) and depending on the symmetry of the electric field gradient at the nuclei (Figure 1), which is defined in equation (1) [5].

$$
\eta=\frac{\frac{\partial^{2} V}{\partial x^{2}}-\frac{\partial^{2} V}{\partial y^{2}}}{\frac{\partial^{2} V}{\partial z^{2}}}
$$

Where $V$ is the electric field gradient at the nuclei and $\mathrm{x}, \mathrm{y}$ and $\mathrm{z}$ are the Cartesian coordinates. Additionally, some molecules have multiple nitrogen atoms with nonequivalent locations that may experience different electric field gradients and have different excitation frequencies. Finally, some compounds exhibit more than one crystallographic form with very different crystalline structure.
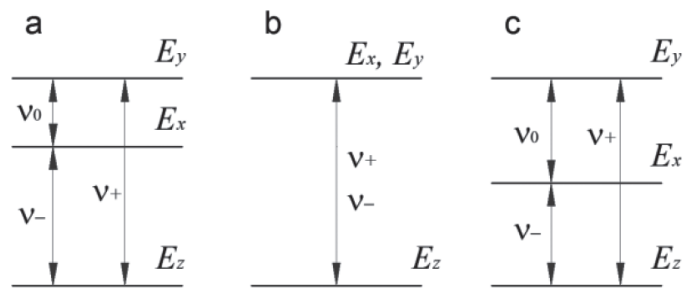

Figure 1. NQR transition frequencies $\left(v_{0}, v_{-}, v_{+}\right)$ between energy levels $\left(E_{x}, E_{y}, E_{z}\right)$ for spin 1 nuclei. In a) $\eta \neq 0$ and all frequencies are different; in b) $\eta=0$ and there is only one frequency $v_{-}=v_{+}$, in c) $\eta=1$ and there are two different frequencies.

Explosive detection by NQR consists of applying a series of RF pulses at the resonant frequency of the explosive (one of the possible frequencies of excitation) and the presence or absence of a return signal is detected. Frequently, the same coil is used for excitation and detection, where the coil operates almost entirely as a magnetometer due to the rapid attenuation of the signal with distance.

For a theoretical analysis of NQR, [6] is recommended. There, a tool was developed to predict NQR dynamics of nuclei having spin 1 . The tool involves only $2 \times 2$ matrix products and can be adapted to each of the NQR transitions. 
In explosive detection there are two possible configurations for an NQR system, one in which the sample is located within the excitation coil and another (remote detection), in which the sample is separated from the coil. The first has application in the detection of explosives in objects that can be placed inside a coil, such as luggage. By contrast, the mine detection can only be made remotely.

When it is used for landmine detection, NQR has the main advantage of having high chemical and crystallographic specificity [2]. Over 50,000 compounds have been investigated and no interference occurs for mine detection [7]. Also, at NQR frequencies (1-5 MHz), RF fields are not attenuated significantly, even in wet soils [8].

\section{NQR DRAWBACKS}

Despite all the advantages of NQR, it has some features that limit its performance. The following describe each of them.

\section{Spin-spin relaxation time}

When an RF pulse is sent to excite ${ }^{14} \mathrm{~N}$, the response is a signal at the same frequency but with exponential decay, called FID (Free Induction Decay), as seen in Figure 2. The rate at which the signal falls is determined by a factor known as transverse relaxation time or spin-spin relaxation time $\left(T_{2}^{*}\right)$. The signal amplitude is given by equation (2).

$$
S_{F I D}(t)=V_{s}\left\langle A_{z}\left(t_{p}\right)\right\rangle e^{-t / T_{2}^{*}}
$$

Where $V_{s}$ is the sample volume and $\left\langle A_{z}\left(t_{p}\right)\right\rangle$ is the response signal to the applied pulse and is a sine function that depends on pulse duration $t_{p}$.

$T_{2}^{*}$ Can be very small depending on the compound (Table 1) and it is related to the line width of the NQR signal spectrum (Figure 3).

As NQR signal is only available for a short time, coil ringdown should be fast enough for the signal to be detectable when the coil is recovered. This requires to employ damping circuits to quickly dissipate coil energy.

\section{Signal strength and noise}

$\mathrm{NQR}$ signal strength is proportional to frequency and as many explosives have excitation frequencies
Table 1. Relaxation times of some explosives at room temperature.

\begin{tabular}{|l|c|c|c|}
\hline Compound & RDX & TNT & AN \\
\hline Frequency & $\mathbf{5 . 1 9} \mathbf{~ M H z}$ & $\mathbf{8 4 2} \mathbf{~ k H z}$ & $\mathbf{4 2 4} \mathbf{~ k H z}$ \\
\hline$T_{1}$ & $11 \mathrm{~ms}$ & $4 \mathrm{~s}$ & $16 \mathrm{~s}$ \\
\hline$T_{2}^{*}$ & $0.8 \mathrm{~ms}$ & $0.4 \mathrm{~ms}$ & $6 \mathrm{~ms}$ \\
\hline$T_{2 e}$ & $16 \mathrm{~ms}$ & $30 \mathrm{~ms}$ & $3 \mathrm{~s}$ \\
\hline $2 \tau$ & $\begin{array}{c}0.7 \mathrm{~ms} \\
{[18]}\end{array}$ & $\begin{array}{c}1.5 \mathrm{~ms} \\
{[55]}\end{array}$ & $\begin{array}{c}2.5 \mathrm{~ms} \\
{[55]}\end{array}$ \\
\hline
\end{tabular}

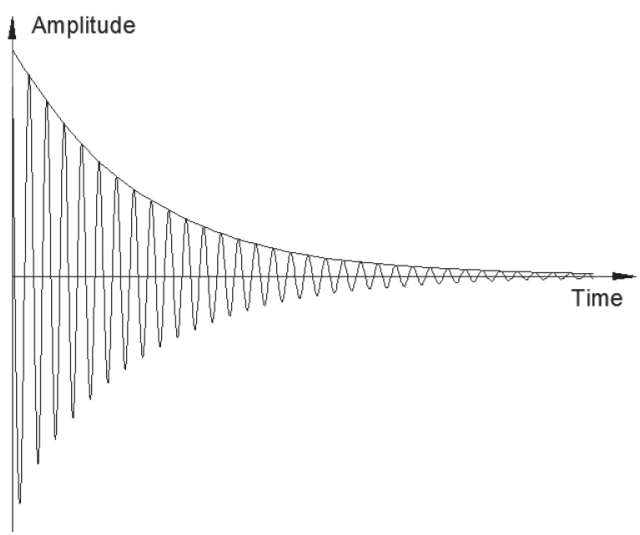

Figure 2. FID signal decays with the time constant $T_{2}^{*}$, which is inverse to the line width.

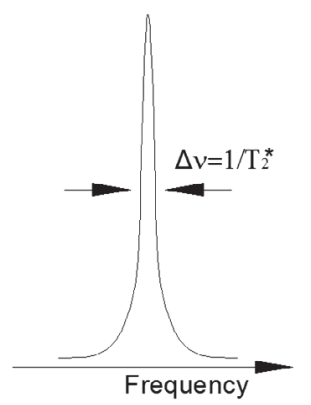

Figure 3. Relationship between line width of NQR signal spectrum and $T_{2}^{*}$.

below $1 \mathrm{MHz}$, the signal is very weak. Thermal random noise of the receiver coil and in a lesser extent, of the preamplifier, limits the signal to noise ratio (SNR) [9]. SNR is also affected by near electromagnetic noise sources [10].

\section{Spin-lattice relaxation time}

To increase SNR, the excitation process may be repeated $n$ times. This way, SNR increases in proportion to $\sqrt{n}$. The problem is that in order to 
perform a second excitation, it is required to wait a time around $5 T_{1}$ to allow fully relaxation of the system, where $T_{1}$ is called longitudinal relaxation time or spin-lattice relaxation time and its value is characteristic of each compound (Table 1). This time is very high for ammonium nitrate (AN) and TNT [11].

\section{Temperature uncertainties}

Temperature has an effect on molecular kinematic, affecting the electric field gradient at the nuclei and likewise the excitation frequencies [12]. For mine detection, estimating explosive temperature is difficult and therefore excitation frequency will not be exactly at resonance and the signal strength will be lesser [13]. To achieve an acceptable result, the temperature of the explosive must be estimated within $\pm 5^{\circ} \mathrm{C}$ precision, which is still difficult to accomplish.

\section{Powdered samples}

Usually, in landmines the explosive is powdered. In powder crystals, orientation of RF field varies from one micro-crystal to another, generating excitation differences between crystals. This equates to reducing signal intensity by $57 \%$ in comparison to a single crystal [9].

\section{Radiofrequency interference (RFI)}

Typical sources of RFI are AM radio transmitters and nearby power sources [14]. Since NQR frequencies of some explosives are within the AM radio band, RFI can completely hide NQR signal. Cancellation of RFI by employing a sensitive antenna to pick up signals from remote RF sources and a suitable algorithm to subtract these signals from NQR channel, is currently the most efficient RFI cancelation approach [15].

\section{Quality factor of the detection system}

The detection system design is another variable significantly affecting NQR performance. Because the signal is too weak to detect, detection systems should have high quality factor $\mathrm{Q}$, as this improves sensitivity. However by increasing Q, transient processes (coil ringdown) take longer times [16]. At low frequencies, the length of the transient processes can reach hundreds of microseconds, making it more difficult to detect compounds with short transverse relaxation times $T_{2}^{*}$ [17].

\section{Polymorphs}

Some chemical compounds, like TNT, form different crystalline structures known as polymorphs and each one can have different excitation frequencies. Because of that, the amount of nuclei that are excited by a RF pulse is less than when there is only one polymorph.

\section{MAPER}

NQR is subject to false alarms due to effects of magneto-acoustic and piezoelectric ringing (MAPER). Piezoelectric materials generate signals at the same NQR excitation frequency while metals generate magneto-acoustic signals.

\section{SOLUTIONS TO NQR DRAWBACKS}

To address the problems mentioned in the previous section, several alternatives have been proposed and they are detailed below.

\section{Multipulse excitation sequences}

To improve the SNR per unit time, multipulse excitation sequences have been proposed. These sequences consist of multiple RF pulses that allow obtaining a signal that repeats after each pulse. Multipulse sequences are of great interest because they improve the detectability of NQR signals as the individual signals (occurring after each pulse) can be added coherently [18]. Multipulse techniques can be divided into two groups: steady-state free precession (SSFP) and spin-lock multipulse (SLMP) [17].

SSFP. The main feature of SSFP sequences is the creation of a steady state continuous signal that lasts as much as the sequence applied [19]. To achieve a strong steady state signal, the repetition time of the pulses in the sequence must be less than or close to $T_{2}^{*}$. The simplest version of the SSFP pulse is represented as $\left(\tau-\theta_{0^{\circ}}^{1}-\tau\right)_{N}$, where $2 \tau$ is the time between pulses, $\theta^{1}$ is the flip angle, $0^{\circ}$ is the pulse phase and $N$ the number of times the sequence is repeated. $\theta$ is related to the change on nuclei orientation and is a function of the RF field strength and pulse duration. SSFP sequences are very effective for detection of explosives with short $T_{1}$, such as RDX.

SLMP. SLMP sequences are based on using the spin-echo effect and are commonly used for detection of explosives with long $T_{1}$, such as AN or TNT. 
Spin echoes occur because the NQR signal decays with the emanations from individual nuclei losing sync. By using special excitation sequences, the resonating nuclei are re-sync and one echo is formed [3]. The basic SLMP sequence can be represented as $\theta_{90^{\circ}}^{0}-\left(\tau-\theta_{0^{\circ}}^{1}-\tau\right)$, where $\theta^{0}$ is the flip angle of the preparatory pulse and $\theta^{1}$ is the flip angle of the other pulses in the sequence. The phase of $\theta^{1}$ is rotated $90^{\circ}$ from the phase of $\theta^{0}$. Figure 4 shows the effect produced by SLMP: after each pulse an echo signal is obtained and the overall amplitude decays with time constant $T_{2 e}$ which is longer than $T_{2}^{*}$ [18] (see Table 1).

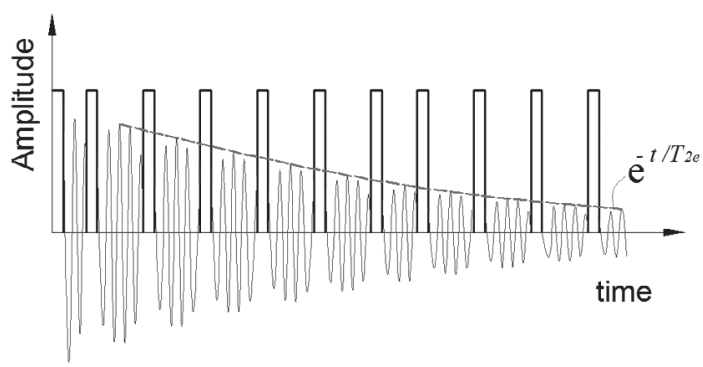

Figure 4. NQR response to SLMP sequence.

The maximum signal amplitude obtained with SLSE sequence is almost two times higher than with the SSFP sequence [19]. Other sequences that combine the properties of both SSFP and SLMP sequences may also be used and in many cases this technique gives better results [17].

In multipulse sequences, the signal amplitude increases with decreasing repetition time $(2 \tau)$. However, the repetition time of the pulses must include the acquisition time and the duration of the coil ringdown. For a proper selection of the sequence parameters, a joint optimization from a derived formula that relates these parameters with the SNR is proposed in [20].

Cycled phase pulses. A mayor problem that arises when developing NQR equipment is to cancel MAPER, generated by numerous objects containing ferrite, metallic, piezoelectric and ferrous components [19]. MAPER have longer life than an RF excitation pulse and its phase is equal to the phase of this pulse (for this reason it is also called coherent noise). This fact is exploited in multipulse sequences to reduce the effect caused by MAPER
[17]. A simple version of the SLMP sequence for coherent noise suppression is:

$$
\theta_{90^{\circ}}^{0}-\left(\tau-\theta_{0^{\circ}}^{1}-\tau-\right)_{N}-\tau-\theta_{\phi}-\tau-\left(\tau-\theta_{0^{\circ}}^{1}-\tau-\right)_{N},
$$

Where $\theta_{\varphi}$ is the flip angle of the intermediate pulse and $\varphi$ is its phase. With this sequence, NQR signal is detected in both blocks of pulses, but the intermediate pulse inverts the phase of the signal (while phase of MAPER is not inverted). Therefore, by subtracting the two blocks, coherent noise can be removed.

Another approach for MAPER suppression consists on applying a sequence block intended to excite NQR signal and MAPER together and a second one to excite only MAPER and then a subtraction is performed. To avoid detection of NQR signals in the second block, a delay is required between the two blocks of pulses and/or the application of a spin saturation technique [17]. The following example of such a sequence is:

$\theta_{90^{\circ}}^{0}-\left(\tau-\theta_{0^{\circ}}^{1}-\tau-\right)_{N}-\left(\tau_{1}-\theta_{\phi}-\tau_{1}-\right)_{n}-\left(\tau-\theta_{0^{\circ}}^{1}-\right.$ $\tau-)_{N}$.

It has been found that the intermediate part results in a substantial loss of NQR signal in the last block, becoming completely eliminated when $\varphi=90^{\circ}$ or $270^{\circ}$. The main disadvantage of this sequence is that in the last block NQR signal is not detected, it is only used to cancel interference signals and therefore SNR per unit time is lower.

A problem with pulse sequences designed to reduce MAPER is that the sequences must be separated by time intervals of the order of $T_{1}$, which means a slower detection. Furthermore, in that period of time, material properties can change by heating due to the excitation. Also, these sequences reduce the NQR signal as a result of destructive interference between spin echoes [21]. These losses are mainly an effect of the averaging of multiple crystals that form a powdered sample. This effect is similar to the problem of inhomogeneous fields in magnetic resonance, a problem that has been addressed by using composite or adiabatic pulses. 
Composite and adiabatic pulses. Composite pulses consist of several back-to-back rectangular pulses with variations in phase, amplitude and/or duration. Adiabatic pulses are pulses with continuous frequency variations [21]. Theoretically, these pulses can produce uniform excitation, resulting in a $15 \%$ improvement in the SNR in powder samples.

In [22], quaternions were proposed for composite pulse design and it was theoretically and experimentally demonstrated that by using quaternions, consecutive rotations applied to the same transition frequency can be described as a single rotation in the space of fictitious spin $1 / 2$ operators. Additionally, by using quaternions to design composite pulses, signals whose amplitude exceeded theoretical predictions in a large percentage were obtained.

In [23] the advantages of using adiabatic half passage (AHP) was demonstrated for NQR detection when applied in powered samples. AHP starts with a RF field excitation far away from the resonance frequency, sweeping the frequency of the RF field until the resonance condition is reached. Experiments with AHP as a preparatory pulse in SLMP sequences demonstrated an almost constant signal amplitude over a rank of RF variations. For NQR detection with inhomogeneous RF fields, as those created by surface coils in landmine detection, AHP advantages can be significant.

Off resonance effects. In pulse sequences, the amplitude of NQR signal varies as a function of resonance frequency offset and time interval between pulses [17]. This effect is known as intensity variations.

In [13], NQR signal dependence on frequency offset was studied, which is related to temperature uncertainties. Results indicate that signal strength and effective relaxation time $T_{2 e}$ vary substantially with frequency offset, showing a periodic variation modulated by the acquisition time $\left(t_{c}\right)$ of the pulse sequence (Figure 5).

In [24] a theory was presented that explains the above observations and includes homonuclear dipolar interactions and spin-lattice coupling. It was shown that spin-lattice coupling is the dominant mechanism of magnetization decay.
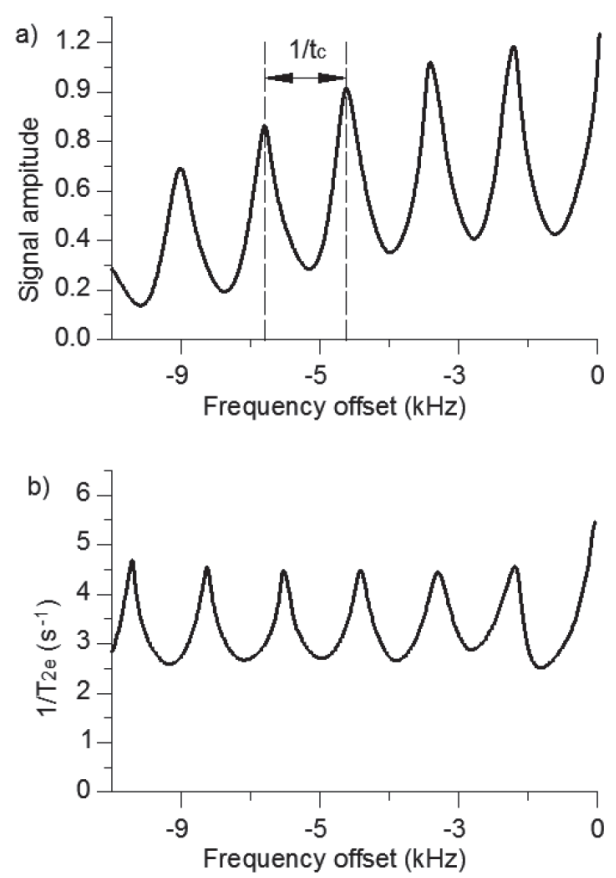

Figure 5. a) Amplitude and b) time constant variations with frequency offset. This is a graphic reproduction of the results in [13] for paranitrotoluene (PNT).

\section{Signal processing algorithms}

To enhance NQR detectability different processing algorithms have been proposed:

In [25] a feedback algorithm that automatically adjusts NQR pulse parameters to increase SNR was experimentally shown. In the proposed method, the measurements of NQR response (specifically the signal power) between RF pulses were used to optimize pulse parameters (pulse duration and frequency of excitation) in real time. The tests were conducted with a sample of $70 \mathrm{~g}$ of sodium nitrite at room temperature (detection was not remote). In [26] the same feedback control methodology was suggested for adjusting pulse parameters, but it was proposed to make the tuning in a pre-screening stage, in which NQR equipment is placed over a test mine so that the algorithm finds optimal pulse parameters for the explosive material, depth, size and environmental conditions. In [27] a pulsed NQR spectrometer is described, designed specifically to facilitate real-time tuning of the pulse sequence parameters. 
Although by using fixed pulse parameters the detected signal strength is sacrificed, tests in [2527] were made under controlled conditions in the absence of MAPER. The presence of such signals could degrade the performance of the control system since they are added to the signal power that is used for feedback.

In [4] an adaptive filter is used to cancel the RFI measured with auxiliary antennas. They propose three detectors: the first two, based on the average power, are a periodogram estimator and MUSIC (Multiple Signal Characterization) and the third is a Bayesian detector. They use data collected by Quantum Magnetics, Inc. for buried mines with TNT and conclude that average power detector using MUSIC performs better than the Bayesian detector because statistic assumptions are not stationary.

In [28] various algorithms where compared to estimate power spectrum of NQR signals. Once the power spectrum is estimated, the detection variable is the integral over a predetermined interval where it is expected to find the NQR signal. The algorithms compared in this paper are: Discrete Fourier Transform (DFT) periodogram, MUSIC and AR (autoregressive spectral estimation) using four ways of estimating the AR parameters: YuleWalker, covariance, modified covariance and Burg. The comparison allows concluding that the modified covariance based detector has better performance. Tests were performed using simulated and real data obtained in a shielded environment, using $180 \mathrm{~g}$ of TNT inside the coil.

In [29] an approximate maximum likelihood (AML) detector was developed that exploits temperature dependence of resonant frequencies, looking for a match between measured data and a model output over an expected temperature and damping constant rank, finding the parameters that give the best match and using them as detection variables. AML detector works over the whole frequency rank, being sensitive to narrowband RFI, like radio transmissions. To reduce this sensitivity, a frequency selective AML detector (FSAML) was introduced in [30] operating on a subset of available frequencies, being robust to narrowband RFI. Also, because of dimensionality reduction of the algorithm, it is computationally more efficient tan AML. AML and FSAML work over the signal resulting of adding the echoes obtained with a multipulse sequence and because of that they operate over that resultant isolated echo and do not exploit the decay of the entire echo train. In [31] AML and FSAML detectors were extended to work over the whole echo train, calling the new detectors ETAML (echo train AML) and FETAML (frequency selective ETAML). Experimental results using from $180 \mathrm{~g}$ of TNT inside a coil show superior performance of the new developed algorithms. However, a problem with these new detectors is that echoes cannot be added and in many applications it is necessary to add the echoes to compensate for the low SNR in a reasonable time.

AML, FSAML, ETAML and FETAML detectors assume known complex amplitudes of NQR signal inside a rank, but those amplitudes are subject to some uncertainty. In [32] a set of robust algorithms were developed that allow for uncertainties on those parameters and estimate them. Detectors work over the whole echo train.

In [33] a performance of NQR detection under RFI interference and complex amplitude uncertainties was addressed with an algorithm that estimates an interference subspace, using data from an auxiliary antenna and projects primary data on an orthogonal space to suppress interference effects. Another algorithm makes a pre-whitening using a transform estimated from data. Simulation results show superior performance in comparison to previous algorithms. In [34] the work was extended to detect polymorphs and explosive mixtures.

In [35] an AR process was proposed to estimate background noise corrupting NQR signals and a matched filter as an explosive detector. Numeric simulations, using experimental background data and synthetic NQR data (generated for RDX), show excellent performance.

In [36] a singular value decomposition (SVD) based on the Hankel matrix is proposed to detect NQR signals. The original signal is decomposed into a superposition of component signals and MUSIC is applied to estimate the power spectrum of each component signal, cancelling RFI and noise by using previous knowledge about NQR frequency. Experiments (using AN inside the detection coil in a shielded environment), show a performance enhancement. 
In [37] a NQR signal processing method is presented based in a Multi stage Wiener Filter (MSWF). MSWF is a reduced rank algorithm implementing Wiener filter using a nested chain of scalar Wiener filters. MSWF does not need a matrix inversion, reducing computational load. Experimental results using AN show that the proposed method effectively reduces RFI and noise in real time.

\section{Superconductors and optical magnetometers}

To improve detectability of NQR signals, some alternatives have been proposed to conventional detection coil.

In [38] a tunable optical magnetometer was developed to detect NQR signals at room temperature. NQR signal from $22 \mathrm{~g}$. of powdered $\mathrm{AN}$ at $2 \mathrm{~cm}$ from the sensor head was obtained with SNR of 9, representing an improvement over coil detection. This work shows that an optical non-cryogenic magnetometer with frequencyindependent sensitivity can be a new attractive tool for NQR detection. However, something that is not mentioned in this paper is the effect of temperature uncertainties. In the experiments, the sample was at stable controlled temperature, but if temperature uncertainties were present, NQR signal could have a frequency that differs from the one at which the magnetometer is tuned, resulting in performance degradation.

In [39] NQR signal was successfully detected at the $888 \mathrm{kHz}$ of PNT using RF SQUID (high Tc RF superconducting quantum interference device) with a normal metallic resonant transformer consisting in a pick-up coil, a smaller input coil and a tuning capacitor. Results show a SNR comparable to the SNR obtained with regular coils and low noise preamplifiers. Authors indicate that by using better coils and switches, SNR could be increased.

In [40], superconductive-magnetoresistive sensors were used to detect NQR signals. The results show that hybrid sensor sensitivity at $800 \mathrm{KHz}$ is comparable to resonant coil sensitivity, but hybrid sensor can be used to simultaneously detect various explosives because they do not have resonant systems.

In [16] "super-Q" detection is studied and results show that SNR behavior depends on frequency offset and preamplifier noise. In a general case, when the preamplifier noise is not zero and excitation is not on resonance, SNR increases with Q until reaching a maximum and then decreases and stabilizes in a constant value. This result is a consequence of the fact that when excitation is off-resonance, signal could be out of detector bandwidth which is pretty narrower at higher values of $\mathrm{Q}$. The author also explores the idea of using Q-switching, i.e. systems having high value of $\mathrm{Q}$ when acquiring the signal and a lower damped value of $\mathrm{Q}$ during (and just after) excitation. He showed results that prove an increase in SNR using Q-switching.

In [14], a CMOS sensor is proposed to detect NQR signals. They also use a small squares adaptive filter to deal with RFI in the analogue domain, before making data digitalization and processing. With the proposed circuitry, requirements for high acquisition accuracy and complex processing algorithms could be relax. They present only simulation results.

\section{Excitation at two resonance frequencies}

NQR excitation at two resonance frequencies causes a signal at the third no irradiated transition. Also, by two frequency excitation, signal intensity at one of the irradiated frequencies can be enhanced [41].

In [42] multi-frequency NQR signals were studied for composite pulse irradiation at two ${ }^{14} \mathrm{~N}$ transitions. Experiments were made with RDX at room temperature using a composite pulse at the second transition as a preparatory pulse on a SLMP sequence. They obtained an increased SNR at one of the excited frequencies, although it was lesser than theory predictions.

In [43] a multi frequency detection method is proposed to detect explosives by NQR, involving $n$ excitation sets where each one consists of a pulse at $v_{-}$, a second pulse at $v_{0}$ and a third pulse at $v_{-}$. NQR signal is detected at $v_{+}$. They showed that the phase of the detected signal depends only on the phase of the excitation at $v_{-}$, which was employed to cancel MAPER. The main advantage of this method is the possibility of avoiding acquisition delays because of coil ringdown. However, it has a drawback: a complex system of three orthogonal coils tuned at three frequencies is required as well as special control systems and complex signal analysis. Also, temperature uncertainties affect differently to each frequency, adding complexity. 


\section{Cross polarization and double resonance}

Double resonance uses ${ }^{1} \mathrm{H}$ nuclei to enhance ${ }^{14} \mathrm{~N}$ signal by polarizing ${ }^{1} \mathrm{H}$ nuclei in a strong magnetic field and transferring this polarization to ${ }^{14} \mathrm{~N}$ nuclei by the crossing of energy levels when the magnetic field is being reduced. In [44] this technique was employed using a weak magnetic field and it was shown a significant increase in ${ }^{14} \mathrm{~N}$ signal and a decrease in $T_{1}$ for TNT by ${ }^{1} \mathrm{H}$ interaction with ${ }^{14} \mathrm{~N}$ transitions.

However, cross polarization techniques require a strong and homogeneous magnetic field, increasing complexity [45] and preventing its use for remote detection. Also, some landmines can be detonated by strong magnetic fields [46].

\section{NQR FOR LANDMINE DETECTION}

Landmine detection by NQR has additional challenges: detection is remote, RFI could be very strong in open environments and there is a coupling between the surface coil and the soil that should be considered.

Because SNR of NQR signal increases by increasing measurement repetitions, it is said that NQR can clear its own false alarms and because of this, NQR has been explored primarily as a confirmation system to solve another sensor alarms [10]. As a confirmation system, NQR could replace prodding, improving demining speed and safety [15].

Literature of NQR for landmine detection comprises the following works:

[47]: The author reports first year results of a landmine detection project with NQR. He used a lower coil for NQR excitation and detection and an identical upper coil to cancel RFI. He made blind tests (where mine size and depth were unknown) with $30 \mathrm{~g}$ and $1 \mathrm{Kg}$ of RDX at three depths inside tubes with dry and wet soil (with clay, sand or organic matter). Scanning time was two seconds. Results showed a detection probability of $90 \%$ with $10 \%$ probability of false alarm. It was also found that soil moisture and texture did not have a systematic effect on the results, the dominant effect was RFI.

[48]: A description of developed NQR technology is described, showing results obtained in blind tests made Fort Leonard Wood, Missouri - U.S. Army. Plastic and metallic landmines with RDX and TNT were detected, employing scanning times of $3 \mathrm{~s}$ when looking for RDX and $16 \mathrm{~s}$ when looking for TNT. They use multipulse sequences and sum echo signals. Power spectrum was compared with a threshold to make the detection. Landmine depth is not considered in the paper. To cancel RFI, authors recommend using auxiliary antennas and an adaptive algorithm instead of a gradiometer.

[9]: A portable sensor was developed and used for landmine detection with this methodology: sensor is placed over an area and a sequence to detect RDX is started, lasting $1 \mathrm{~s}$. Then, the same procedure is executed for TNT lasting $0.6 \mathrm{~s}$. As it was important to maintain scanning time below $2 \mathrm{~s}$, only one SLMP sequence repetition was employed for TNT. Scanning time at each place lasts $1.5 \mathrm{~s}$, but data storing, processing and control sum another $12 \mathrm{~s}$. Detection is based on signal intensity. $100 \%$ probability of detection for TNT was reached, but with a high amount of false alarms (14 alarms). But it was showed that by repeating the whole sequence another time, false alarms could be suppressed.

[49]: A NQR detector was developed and remote measurements were made, comparing results for various sensor-sample separation distances. Tests were also made using and not using a ferrite bar inside the detection coil to increase sensitivity. Results indicate that the use of the ferrite bar triple signal intensity. Using a $100 \mathrm{~W}$ power amplifier and a scanning time of 1.7 minutes, they detected landmines at $2.5 \mathrm{~cm}$ from the sensor. Detection is based on signal intensity.

[45]: A NQR prototype is described and results are presented for sodium nitrite detection. They used SLMP sequence for excitation and showed that scanning times required for detection increase with mine depth ( $3 \mathrm{~s}$ for $3 \mathrm{~cm}$ depth and $90 \mathrm{~s}$ for 10 $\mathrm{cm}$ depth). Detection is based on signal spectrum, finding the highest peak and looking if it is inside an expected interval.

In [50] an NQR system was developed to detect RDX. Tests were made at different mine depths for $100 \mathrm{~g}$ of RDX. System takes 13 minutes per scan. Under these conditions they were capable of detecting RDX into a landmine at $15 \mathrm{~cm}$ depth and 
outside a shielded room. They also made tests with a lower detection time (two minutes per scan) and for this time, the detection limit was $100 \mathrm{~g}$ of RDX at $10 \mathrm{~cm}$. Detection was based on signal strength.

\section{AMMONIUM NITRATE (AN)}

Something usual in landmine detection literature is that American countries are rarely mentioned as being affected by landmines. Instead, Cambodia, Afghanistan, Croatia and Angola are the most included places for research. However, landmine problem is present in some American countries, being Colombia the most affected and ranking second in the world according to the number of victims [51]. Another thing that is not mentioned in landmine detection literature is that in some countries, like Colombia, most antipersonnel landmines are homemade using ANFO (ammonium nitrate and fuel oil) [52]. For this reason, AN detection by $\mathrm{NQR}$ is included on this review.

AN has four structural transitions at atmospheric pressure [53]: The stable polymorph at room temperature (from $16^{\circ} \mathrm{C}$ to $32^{\circ} \mathrm{C}$ ) is the IV. An AN crystal consists of ammonium and nitrate ions linked by $\mathrm{N}-\mathrm{H}-\mathrm{O}$ bonds. Excitation frequencies for ${ }^{14} \mathrm{~N}$ nuclei at nitrate ion are higher than those for ammonium ion. Some parameters of frequencies $v_{+}$ and $v_{-}$of nitrate ion, which are of most interest for being the higher ones, are presented in Table 2 [54].

Table 2. Parameters for transition frequencies $v_{+}$ and $v_{-}$for nitrate ion at ammonium nitrate molecule [54].

\begin{tabular}{|l|c|c|}
\hline Line & $v_{+}$ & $v_{-}$ \\
\hline Frequency $(\mathrm{kHz})$ & 496.4 & 423.4 \\
\hline Temperature coefficient $\left(\mathrm{Hz} /{ }^{\circ} \mathrm{K}\right)$ & -300 & +91 \\
\hline $\begin{array}{l}\text { Temperature coefficient for } T_{1} \\
\left(\mathrm{~s} /{ }^{\circ} \mathrm{K}\right)\end{array}$ & 0.2 & 0.34 \\
\hline$T_{1}(\mathrm{~s})$ & 13 & 16 \\
\hline$T_{2}(\mathrm{~ms})$ & 8 & 6 \\
\hline $\begin{array}{l}T_{2 e} \text { for } 3 \mathrm{~ms} \text { of pulse interval in } \\
\text { SLMP }\end{array}$ & 3.02 & 2.5 \\
\hline
\end{tabular}

According to [55], NQR for AN detection is very convenient when multipulse excitation sequences are applied because $T_{2 e}$ is pretty long (longer than for TNT) and this way, signal intensity of AN at $424 \mathrm{kHz}$ is two times stronger than that of TNT at $842 \mathrm{kHz}$. In NQR literature there are few studies around $\mathrm{AN}$ detection and any of them is related to the landmine detection problem. Papers that were found on the subject include [53], in which NQR excitation frequencies, polymorphs and relaxation times for AN were measured; [54] where a system for AN detection inside vehicles was developed, in which the vehicle is passed through a big coil (detection is not remote); [56], where double resonance method is used to detect $\mathrm{AN}$ and [57] in which NQR is applied for ANFO and REOGEL+ detection, two explosives made with AN. In this last work, SSFP and SLMP sequences were compared and it was concluded that SLMP sequences have better performance for AN. They also optimized this sequence using composite preparatory and refocusing pulses, achieving a SNR increment of $20 \%$. In this paper, detection is not remote and RFI as well as sample-sensor separation are not taken into account. An important conclusion of this paper is that the various ANFO samples investigated had the same relaxation parameters as pure AN, contrary to what happened with RIOGEL+.

\section{CONCLUSION}

NQR is a spectroscopic technique that allows detecting explosives with high chemical specificity, being suited for landmine detection.

There are various factors affecting the obtained SNR: molecular relaxation parameters, RFI, thermal noise, sample-sensor distance and MAPER. In NQR literature for explosives detection, researches are found looking to solve one or more of such problems, but most of them have only been tested in simulation or with data obtained under shielded laboratory conditions where detection is not remote and this way the performance of those developments in landmine detection is uncertain. On the other side, landmine detection research has been focused on using this technique as a confirmation system for the alarms given by another sensor. As SNR increases with scanning time, those works have used signal power around the expected frequency as a detection variable, caring for scanning long enough to get a high confidence (high SNR). On this way, there is a perceived gap between NQR literature for explosives detection and for landmine detection, since many contributions in NQR literature have not been applied to the landmine detection problem. 
Also, landmine detection by NQR is focused on military explosives (RDX, TNT and Tetryl) and research on detection AN is not related to landmine detection, which is lamentable given the fact that in some countries, like Colombia (the most affected country in America by the landmine problem and the second in the world), $\mathrm{AN}$ is the most common explosive in landmines.

In the literature, few works are found that use NQR as a landmine detection system instead of as an alarm solving system because conventional landmines are mostly filled with TNT and its NQR parameters make the detection task hard to achieve in a reasonable time

Although NQR as confirmation system is an important contribution to the demining problem, having significant advantages over prodding and work in the area is the result of significant efforts, it does not mean that NQR cannot be thought as a detection system, especially for AN, since the NQR signal for this compound last longer than TNT signal when multipulse sequences are used and the excitation frequency is not inside the AM radio band [55].

On the other side, according to [47], RFI was the main factor affecting landmine detection performance and making the analogy that RFI and MAPER are to $\mathrm{NQR}$ as clutter is to ground penetrating radar (GPR) [10], solutions to clutter in GPR literature can be extrapolated to NQR. In most GPR literature landmine detection is seen as a two categories classification task: mine class and no mine class (i.e. clutter class) and classifiers are trained using data from the two classes. Similarly, this vision could be applied to NQR, registering data with and without mines and using these data to train a classifier. Stating the problem in this way opens the possibility to a fusion of NQR and another technology, such as GPR, at the feature level. This is important, since from the landmine detection literature, a general conclusion is that a sensor fusion is needed to solve the problem [7,58-59].

Active cancellation of RFI by using auxiliary antennas and multipulse sequences (SSFP and SLMP) with alternated phases to cancel MAPER are already used in landmine detection literature by NQR. However, there are another works that have not been explored in the landmine detection problem and between them, these are consider most suited for landmine detection:

- Detection at high values of Q using Q switching, as it was proposed in [16].

- Development of a function relating SNR with multipulse sequence parameters to optimize those parameters, as it was made in [20].

- The use of composite pulses and adiabatic pulses, primary as preparatory pulses to enhance SNR [22-23].

- Testing detection algorithms created to deal with RFI and temperature uncertainties: [30,33,36-37].

- Research in atomic magnetometers and superconducting devices are promising, but more research is needed to apply them in field. In this area, there is a patent of a landmine detection system with SQUID [60].

Finally, it is expected that this review can help in thinking landmine detection by NQR from another perspective and this way, the problem faced by many countries because of landmines could find a solution.

\section{REFERENCES}

[1] H. Kasban, O. Zahran, S.M. Elaraby, M. El-Kordy and F.E. Abd El-Samie. "A comparative study of landmine detection techniques". Sensing and Imaging: An International Journal. Vol. $11 \mathrm{~N}^{\circ} 3$, pp. 89112. July, 2010. ISSN: 1557-2072. DOI: 10.1007/s11220-010-0054-X

[2] Z. Bielecki, J. Janucki, A. Kawalec, J. Mikolajczyk, N. Palka, M. Pasternak, T. Pustelny, T. Stacewicz and J. Wojtas. "Sensors and systems for the detection of explosive devices-an overview". Metrology and Measurement Systems. Vol. XIX, $\mathrm{N}^{\mathrm{o}} 1$, pp. 3-28. March, 2012. ISSN: 0860-8229. DOI: $10.2478 / v 10178-012-0001-3$

[3] J. B. Miller and G. A. Barrall. "Explosives detection with nuclear quadrupole resonance : an emerging technology will help to uncover land mines and terrorist bombs". 
American Scientist. Vol. 93 N $^{\circ}$ 1, pp. 50-57. January, 2005. ISSN: 0003-0996. DOI: 10.1511/2005.1.50.

[4] Y. Tan, S.L. Tantum and L.M. Collins. "Landmine detection with nuclear quadrupole resonance". IEEE Geoscience and Remote Sensing Symposium. Toronto, Canada. 24-28 June, 2002.

[5] Y.K. Lee. "Spin-1 nuclear quadrupole resonance theory with comparisons to nuclear magnetic resonance". Concepts in Magnetic Resonance. Vol. $14 \mathrm{~N}^{\circ}$ 3, pp. 155-171. April, 2002. ISSN: 1552-5023. DOI: 10.1002/ cmr. 10023.

[6] D. Canet and M. Ferrari. "Fundamentals of pulsed nitrogen-14 quadrupole resonance". In Explosives Detection Using Magnetic and Nuclear Resonance Techniques. Springer. 1st ed. Dordrecht, The Netherlands, pp. 1-29. 2009. ISBN: 978-90-481-3062-7.

[7] S.W. Kercel, R.S. Burlage, D.R. Patek, C.M. Smith, A.D. Hibbs and T.J. Rayner. "Novel methods for detecting buried explosive devices". Proceedings of SPIE Detection and Remediation Technologies for Mines and Minelike Targets. Orlando, USA. April 21, 1997.

[8] A.N. Garroway. "Nuclear quadrupole resonance (paper ii)". In Alternatives for Landmine Detection. RAND Corporation, 1st ed, pp. 179-189. Santa Monica, CA. 2003. ISBN: 0833033018

[9] A.N. Garroway, M.L. Buess, J.B. Miller, B.H. Suits, A.D. Hibbs, G.A. Barrall, $\mathrm{R}$ Matthews and L.J. Burnett. "Remote sensing by nuclear quadrupole resonance". IEEE Transactions on Geoscience and Remote Sensing. Vol. 39 N $^{\circ}$ 6, pp. 11081118. June, 2001. ISSN: 0196-2892. DOI: $10.1109 / 36.927420$.

[10] A.D. Hibbs. "Nuclear quadrupole resonance (paper i)". In Alternatives for Landmine Detection. RAND Corporation, 1st ed, pp. 169-178. Santa Monica, CA. 2003. ISBN: 0833033018.

[11] E. Gudmundson, A. Jakobsson and P. Stoica. "NQR-based explosives detectionan overview". International Symposium on Signals, Circuits and Systems. Lasi, Romania. July 9-10, 2009.
[12] R.M. Deas, C. Cervantes and S.F. Schaedel. "Landmine detection by nuclear quadrupole resonance (NQR)". Army communications - electronics commandfort belvoir va night vision and electronics sensors directorate, 2004.

[13] A. Gregorovič, T. Apih, J. LuŽnik, J. Pirnat and Z. Trontelj. " $14 \mathrm{~N}$ nuclear quadrupole resonance signals in paranitrotoluene and trinitrotoluene : Spin-lock spin-echo offresonance effects". In Explosives Detection Using Magnetic and Nuclear Resonance Techniques. Springer Netherlands. 1st ed. Dordrecht, The Netherlands, pp. 171-191. 2009. ISBN: 978-90-481-3062-7.

[14] X. Zhang, S. Balk, M.W. Hoffman and N. Schemm. "A robust CMOS receiver frontend for nuclear quadrupole resonance based explosives detection". 53rd IEEE International Midwest Symposium on Circuits and Systems. Seattle, USA. August 1-4, 2010.

[15] P. J. Prado, Y. K. Lee, H. Robert and G. A. Barrall."Landmine detection by quadrupole resonance". in Identification of Mines and Unexploded Ordnance. Harvard University Press. Book editing in progress.

[16] A. Gregorovič and T. Apih. "Aplicability of TNT 'super-Q detection' to multipulse sequences". Journal of magnetic resonance. Vol. $201 \mathrm{~N}^{\circ}$ 2, pp. 131-6. December, 2009. ISSN: 1090-7807. DOI:10.1016/j. jmr.2009.08.013

[17] T.N. Rudakov. "Detection of explosives by NQR method: main aspects for transport security". In Explosives Detection Using Magnetic and Nuclear Resonance Techniques. Springer Netherlands. 1st ed. Dordrecht, The Netherlands, pp. 1-29. 2009. ISBN: 978-90-481-3062-7.

[18] T.N. Rudakov, V.T. Mikhaltsevich and O.P. Selchikhin. "The use of multi-pulse nuclear quadrupole resonance techniques for the detection of explosives containing RDX". Journal of Physics D: Applied Physics. Vol. 30 $\mathrm{N}^{\circ}$ 9, pp. 1377-1382. May, 1997. ISSN: 13616463. DOI: 10.1088/0022-3727/30/9/013.

[19] V.T. Mikhaltsevitch. "Techniques used for 14N NQR studies". In Annual Reports on NMR Spectroscopy. Elsevier Ltd. 1st ed. Oxford, UK. Vol. 66, pp. 149-194. 2009. ISBN: 978-0-12-374737-2. 
[20] A.E. Mefed. "Joint optimization of the number of pulses and the relaxation pause in the combined multiple-pulse spin-locking sequence for detecting explosives by NQR". Applied Magnetic Resonance. Vol. $38 \mathrm{~N}^{\circ} 1$, pp. 51-64. December, 2009. ISSN: 16137507. DOI: 10.1007/s00723-009-0054-y.

[21] M.L.B. Joel B. Miller, Karen L. Sauer and Christopher A. Klug. "Efficient excitation and ringing suppression in nuclear quadrupole resonance". in Explosives Detection Using Magnetic and Nuclear Resonance Techniques. Springer. 1st ed. Dordrecht, The Netherlands, pp. 1-29. 2009. ISBN: 978-90-481-3062-7.

[22] K.L. Sauer, C.A. Klug, J.B. Miller and A. N. Garroway. "Using quaternions to design composite pulses for spin-1 NQR" Applied Magnetic Resonance. Vol. $25 \mathrm{~N}^{\circ} 3-4$, pp. 485500. September, 2004. ISSN: 1613-7507. DOI: $10.1007 / \mathrm{BF} 03166543$.

[23] J.B. Miller and A.N. Garroway. "Applications of adiabatic half passage to NQR". Applied Magnetic Resonance. Vol. $25 \mathrm{~N}^{\circ} 3-4$, pp. 475483. September, 2004. ISSN: 1613-7507. DOI: $10.1007 / \mathrm{BF} 03166542$.

[24] A. Gregorovič and T. Apih. "Relaxation during spin-lock spin-echo pulse sequence in $14 \mathrm{~N}$ nuclear quadrupole resonance". The Journal of chemical physics. Vol. $129 \mathrm{~N}^{\circ} 21$, pp. 214-504, December, 2008. ISSN: 10897690. DOI: $10.1063 / 1.3023091$.

[25] J.L. Schiano, A.J. Blauch and M.D. Ginsberg. "Optimization of NQR pulse parameters using feedback control". XVth International Symposium on Nuclear Quadrupole Interactions. Leipzig, Germany. July 25-30, 1999.

[26] J.L. Schiano, T. Routhier, A. J. Blauch and M.D. Ginsberg. "Feedback optimization of pulse width in the SORC sequence". Journal of magnetic resonance. Vol. 140 $\mathrm{N}^{\circ}$ 1, pp. 84-90. Sep., 1999. ISSN: 10907807. DOI: 10.1006/jmre.1999.1824.

[27] J.L. Schiano and M.D. Ginsberg. "A pulsed spectrometer designed for feedback nqr". XVth International Symposium on Nuclear Quadrupole Interactions. Leipzig, Germany. July 25-30, 1999.

[28] S.D. Somasundaram, K. Althoefer, J.A.S. Smith and L.D. Seneviratne. "Detection of landmines using nuclear quadrupole resonance (NQR): signal processing to aid classification". Proceedings of the 8th International Conference on Climbing and Walking Robots and the Support Technologies for Mobile Machines. London, UK, September 13-15, 2005.

[29] A. Jakobsson, M. Mossberg, M.D. Rowe and J.A.S. Smith. "Exploiting temperature dependency in the detection of NQR signals". IEEE Transactions on Signal Processing. Vol. $54 \mathrm{~N}^{\circ}$ 5, pp. 1610-1616. May, 2006. ISSN: 1053-587X. DOI: 10.1109/TSP.2006.871969.

[30] A. Jakobsson, M. Mossberg, M.D. Rowe and J.A.S. Smith. "Frequency-selective detection of nuclear quadrupole resonance signals". IEEE Transactions on Geoscience and Remote Sensing. Vol. 43 NN$^{\circ} 11$, pp. 26592665, November, 2005. ISSN: 0196-2892. DOI: 10.1109/TGRS.2005.856633.

[31] S.D. Somasundaram, A. Jakobsson, J.A.S. Smith and K. Althoefer. "Exploiting spin echo decay in the detection of nuclear quadrupole resonance signals". IEEE Transactions on Geoscience and Remote Sensing. Vol. 45 $\mathrm{N}^{\circ}$ 4, pp. 925-933. April, 2007. ISSN: 01962892. DOI: 10.1109/TGRS.2006.890413.

[32] S.D. Somasundaram, A. Jakobsson and E. Gudmundson. "Robust nuclear quadrupole resonance signal detection allowing for amplitude uncertainties".IEEE Transactions on Signal Processing. Vol. $56 \mathrm{~N}^{\circ} 3$, pp. 887894, March, 2008. ISSN:1053-587X. DOI:10.1109/TSP.2007.909041.

[33] S.D. Somasundaram, A. Jakobsson and N.R. Butt. "Countering radio frequency interference in single-sensor quadrupole resonance". IEEE Geoscience and Remote Sensing Letters. Vol. $6 \mathrm{~N}^{\circ}$ 1, pp. 62-66. June, 2009. ISSN: 1545-598X. DOI: 10.1109/ LGRS.2008.2005792.

[34] T. Rudberg and A. Jakobsson. "Robust detection of nuclear quadrupole resonance signals in a non-shielded environment". 19th European Signal Processing Conference. Barcelona, Spain. August 29 - September 2, 2011.

[35] J. Niu, T. Su, X. He, K. Zhu and H. Wu. "Weak NQR signal detection based on generalized matched filter". Procedia Engineering. Vol. 7 $\mathrm{N}^{\mathrm{o}} 1$, pp. 377-382. June, 2010. ISSN: $1877-$ 7058. DOI: 10.1016/j.proeng.2010.11.061. 
[36] K. Zhu, T. Su, X. He and J. Niu. "SVD for enhanced explosives detection using NQR". Procedia Engineering. Vol. $7 \mathrm{~N}^{\circ} 1$, pp. 57-62, June, 2010. ISSN: 1877-7058. DOI: 10.1016/j.proeng.2010.11.009.

[37] T. Yang, T. Su and X. He. "NQR signal processing based on multi-stage Wiener filter". Procedia Engineering. Vol. $7 \mathrm{~N}^{\mathrm{o}} 1$, pp. 229-234. Juny, 2010. ISSN: 1877-7058. DOI: 10.1016/j.proeng.2010.11.036.

[38] S.-K. Lee, K.L. Sauer, S.J. Seltzer, O. Alem and M. V. Romalis. "Subfemtotesla radiofrequency atomic magnetometer for detection of nuclear quadrupole resonance" Applied Physics Letters. Vol. $89 \mathrm{~N}^{\circ}$ 21, pp. 214106, November, 2006. ISSN: 1077-3118. DOI: 10.1063/1.2390643.

[39] M. Tachiki, D.F. He and H. Itozaki. "Sensing of chemical substances using SQUID-based nuclear quadrupole resonance". Physica C: Superconductivity. Vol. 463-465, pp. 10341037. October, 2007. ISSN: 0921-4534. DOI: 10.1016/j.physc.2007.01.054.

[40] M. Pannetier-Lecoeur, C. Fermon, H. Dyvorne, G. Cannies and G. Le Goff. "14N NQR detection of explosives with hybrid sensors". In Explosives Detection Using Magnetic and Nuclear Resonance Techniques. Springer Netherlands. 1st ed. Dordrecht, The Netherlands, pp. 31-39. 2009. ISBN: 978-90-481-3062-7.

[41] B.A. George, V. Mozzhukhin, B.Z. Rameev and N. DoĞan. "The twofrequency multipulse sequence in nuclear quadrupole resonance of N-14 nuclei”. In Explosives Detection Using Magnetic and Nuclear Resonance Techniques. Springer Netherlands. 1st ed. Dordrecht, The Netherlands, pp. 205-230. 2009. ISBN: 978-90-481-3062-7.

[42] G.V. Mozzhukhin, B.Z. Rameev, N. Doğan and B. Aktaş. "The application of the two frequency composite pulses for NQR detection of nitrogen-based compounds". Journal of superconductivity and novel magnetism. Vol. $24 \mathrm{~N}^{\circ} 1-2$, pp. 653-658. Juny, 2011. ISSN: 1557-1947. DOI: 10.1007/ s10948-010-0928-0.

[43] G.V. Mozzhukhin, B.Z. Rameev, R.R. Khusnutdinov, N. Doğan and B. Aktas. "Three-frequency composite multipulse nuclear quadrupole resonance technique for explosive detection". Applied Magnetic Resonance. Vol. 43 N $^{\circ}$ 4, pp. 547-556. April, 2012. ISSN: 1613-7507. DOI: $10.1007 /$ s00723-012-0326-9.

[44] K.R. Thurber, K. L. Sauer, M.L. Buess, C. A. Klug and J. B. Miller. "Increasing 14N NQR signal by $1 \mathrm{H}-14 \mathrm{~N}$ level crossing with small magnetic fields". Journal of magnetic resonance. Vol. $177 \mathrm{~N}^{\circ} 1$, pp. 118-28, November, 2005. ISSN: 1090-7807. DOI: 10.1016/j.jmr.2005.07.016.

[45] M. Ostafin and B. Nogaj. "14N-NQR based device for detection of explosives in landmines". Measurement. Vol. $40 \mathrm{~N}^{\mathrm{o}} 1$, pp. 43-54. June, 2007. ISSN: 0263-2241. DOI: 10.1016/j.measurement.2006.04.003.

[46] V.S. Grechishkin, R.V. Grechishkina and H. Heo. "Signal processing methods in NQR". In Explosives Detection Using Magnetic and Nuclear Resonance Techniques. Springer Netherlands. 1st ed. Dordrecht, The Netherlands, pp. 159-170. 2009. ISBN: 978-90-481-3062-7.

[47] A.D. Hibbs, G.A. Barrall, P.V. Czipott, A.J. Drew, D.K. Lathrop, Y.K. Lee, E.E. Magnuson, R. Matthews, D.C. Skvoretz and S.A. Vierkotter. "Man portable mine detector using nuclear quadrupole resonance - first year progress and test results". Second International Conference on the Detection of Abandoned Land Mines. Edinburgh, Scotland. October 12-14, 1998.

[48] A.D. Hibbs. "In-Situ Real Time Detection of Explosive-Chemical Compounds in Mines Using Nuclear Quadrupole Resonance (NQR)”. Quantum Magnetics Inc. San Diego, CA. 2001.

[49] K. Hashi, T. Shimizu, H. Itozaki, M. Tachiki, T. Kondo and K. Kawagishi. "Development of a remote nuclear quadrupole resonance detector". Japanese Journal of Applied Physics. Vol. 42 N$^{\circ}$ 12A, pp. L1481-L1482. Dec. 2003. ISSN: 1347-4065. DOI: 10.1143/ JJAP.42.L1481.

[50] H. Itozaki. "Nuclear quadrupole resonance for explosive detection". in Anti-personnel Landmine detection for humanitarian demining: the current situation and future direction for japanese research and development. K. Springer-Verlag, 1 st. ed. 
London, England, pp. 148-155. 2009. ISBN: 1848823452.

[51] A. EFE. "Colombia, segundo país con mayor número de víctimas de minas". Periódico el País, Cali, Colombia. Seccion Judicial, November 24, 2011.

[52] Y.A. Pino. "Determinación de técnicas de detección de explosivos óptimas para el departamento de Antioquia". Trabajo de grado. Universidad Nacional de Colombia, sede Medellín. 2009.

[53] J. Seliger, V. Zagar and R. Blinc, "14N NQR study of the structural phase transitions in NH4NO3". Zeitschrift für Physik B Condensed Matter. Vol. 77 No 3, pp. 439443. October,1989. ISSN: 0722-3277. DOI: 10.1007/BF01453795.

[54] J. Barras, M.J. Gaskell, N. Hunt, R.I. Jenkinson, K.R. Mann, D.A.G. Pedder, G.N. Shilstone and J.A.S. Smith. "Detection of ammonium nitrate inside vehicles by nuclear quadrupole resonance". Applied Magnetic Resonance. Vol. $25 \mathrm{~N}^{\circ}$ 3-4, pp. 411-437. September, 2004. ISSN: 1613-7507. DOI: 10.1007/BF03166538

[55] G.V. Mozzhukhin, S.V. Molchanov, G.S. Kupriyanova, A.V. Bodnya, V.V. Fedotov, H. Guoxin, J. Yanbo, R. Tianliang and Z. Guojin. "The Detection of industrial explosives by the quadrupole resonance method: some aspects of the detection of ammonium nitrate and trinitrotoluene". In Explosives Detection Using Magnetic and Nuclear Resonance Techniques. Springer Netherlands. 1st ed. Dordrecht, The Netherlands, pp. 231-244. 2009. ISBN: 978-90-481-3062-7.

[56] D.W. Prescott. "Nuclear quadrupole spin dynamics: how weak rf pulses and double resonance cross-relaxation contribute to explosives detection". Doctoral Thesis, George Mason University. 2010.

[57] T.N. Rudakov. "Some aspects of the effective detection of ammonium nitratebased explosives by pulsed NQR method". Applied Magnetic Resonance. Vol. $43 \mathrm{~N}^{\mathrm{o}} 4$, pp. 557-566. April, 2012. ISSN: 1613-7507. DOI: 10.1007/s00723-012-0330-0.

[58] C. King, P. Blagden, G. Rhodes, L. Maresca, A. Wheatley, R. Mann, S. Maslen, I. Mansfield, M. Yarmoshuk and T. Paterson. Mine action : lessons and challenges. Geneva International Centre for Humanitarian Demining. 1st. ed. Geneva, Switerzalnd, pp. 374. 2005. ISBN: 2884870253.

[59] L. Robledo, M. Carrasco and D. Mery. "A survey of land mine detection technology". International Journal of Remote Sensing. Vol. $30 N^{\circ}$ 9, pp. 2399-2410. May, 2009. ISSN: 13665901. DOI: 10.1080/01431160802549435.

[60] H. Itozaki, K. Kawagishi, T. Kondo, T. Shimizu and K. Hashi. "Mine Detector with NQR-SQUID”. U.S. Patent US73942502008. 